\title{
Comparison Study for Three Compression Techniques (Wavelet, Contourlet and Curvelet Transformation)
}

\author{
Shahad M. Sulaiman \\ shahad.csp161@student.uomosul.edu.iq \\ Hadia S. Abdullah \\ hadiasalih@uomosul.edu.iq \\ Department of Computer Science \\ College of Computer Science and Mathematics \\ University of Mosul, Mosul, Iraq
}

Received on: 18/11/2020

Accepted on: 05/01/2021

\begin{abstract}
Researches and studies on compressing digital images are aiming to make it easier to deal with networks, communications and Internet by reducing the size of the multimedia files transferred, and reducing the execution time and transmission time. In this research, the lossy compression method was adopted as one of the solutions that reduce the size of the data required to compress the image, through the process of compression of digital image data using Discrete Wavelet Transform algorithms using Haar filter, and Contourlet. Using Laplace and Directional Filter, Curvelet transformation using FDCT- Wrapping Technology. The performance of the algorithms used in the proposed research is also evaluated using a Ratio Compression (RC) scale, As well as the Peak signal to noise ratio (PSNR) scale, the mean sequence error (MSE) scale, the signal to noise ratio (SNR) scale, and finally, the Normalization correlation (NC) scale. Correspondence between the original image and the recovered image after compression, in order to choose the best algorithm that achieves the best compression ratio of the image and maintains the parameters of the recovered image based on the standards (MSE, PSNR, SNR, COR and CR) used with the three algorithms, and the results showed that the Curvelet transformation algorithm achieved : best compression ratio, but at the expense of image quality.
\end{abstract}

Keywords: Digital Image Compression, Wavelet Transform, Contourlet Transform, Curvelet Transform.

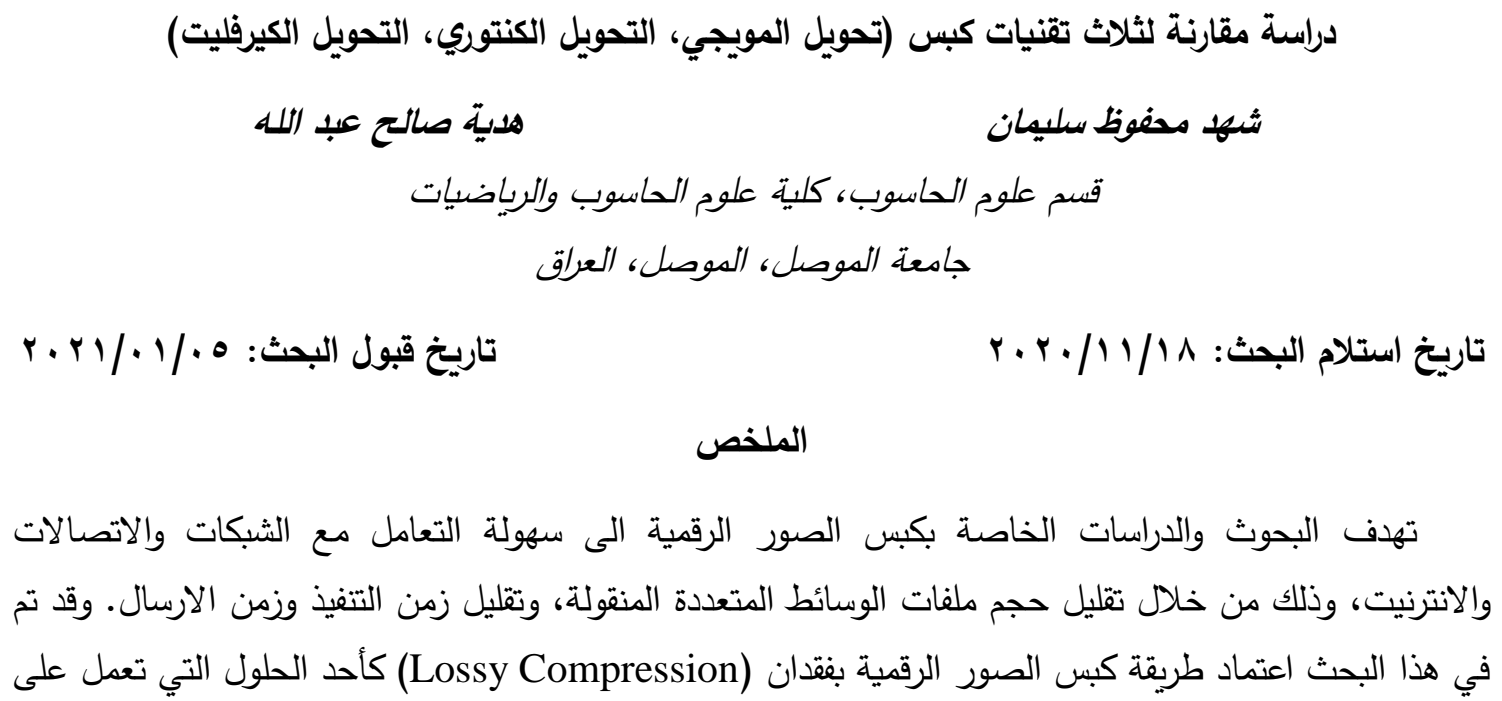


تقليل حجم البيانات المطلوبة لكبس الصورة، وذلك من خلال عملية كبس بيانات الصورة الرقمية باستخدام

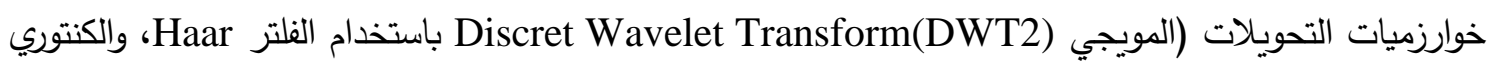
Contourlet Transform باستخدام الفلتر لابلاس والاتجاهي، والكيرفليت Curvelet Transform باستخدام تقنية التغليف (warpping)(. كما يتم تقييم أداء الخوارزميات المستخدمة في البحث المقترح باستخدام مقياس

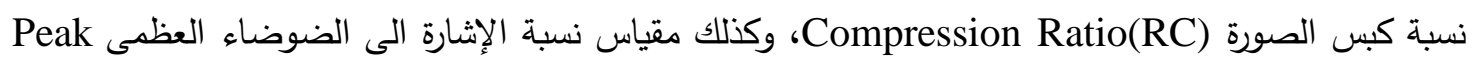
،mean sequence error (MSE) signal to noise ratio(PSNR) ومقياس نسبة الاشارة الى الضوضاء (Signal to noise ratio (SNR) واخيراً مقياس ترابط التربيعي Normalization correlation (NC) الكبس. وذلك لاختيار افضل خوارزمية تحقق افضل نسبة كبس للصورة وتحافظ على معالم الصورة المسترجعة بالاعتماد على مقاييس (MSE, PSNR, SNR, COR and CR) المستخدمة مع الخوارزميات الثلاثة، واظهرت النتائج ان خوارزمية تحويل الكيرفليت حققت افضل نسبة كبس، ولكن على حساب جودة الصورة. الكلمات المفتاحية: كبس الصورة الرقية، التحويل المويجي، التحويل الكنتوري، التحويل الكيرفليت.

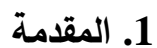

إن مصطلح معالجة الصور الرقمية (Image Processing) هي تمثيل للصور الثنائية الابعاد على الحاسوب وبواسطة الصفر والواحد (01)، وتتكون كل صورة رقمية على الحاسوب من (Pixel) وهو اصغر وحدة في الصورة وكل صورة تحتوي على صفوف واعمدة من البكسلات (pixels) وكلما زادت عدد البكسلات كانت الصورة أوضح [1].

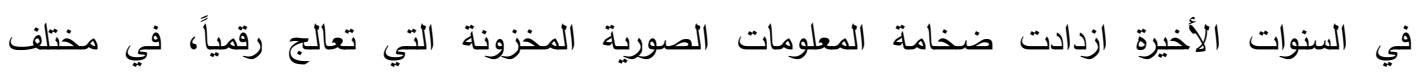
الاختصاصات والتطبيقات ،وان عملية نقل المعلومات عبر الوسائط المتعددة او على شبكة الانترنيت تحتاج الى فئي

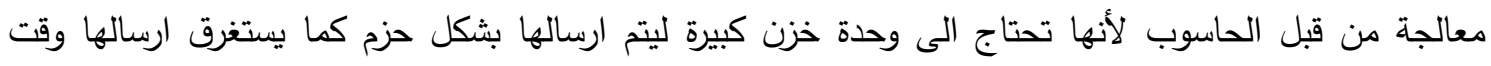
طويل ولهذا اصبحت التقنيات بحاجة الى الكبس [2]. تتضمن عملية الكبس مفهومين اساسيين هما تقليل

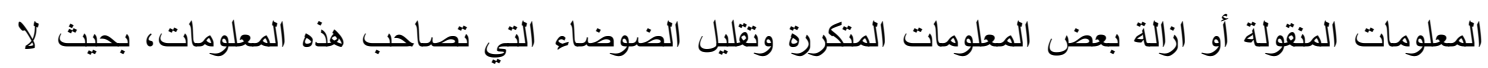

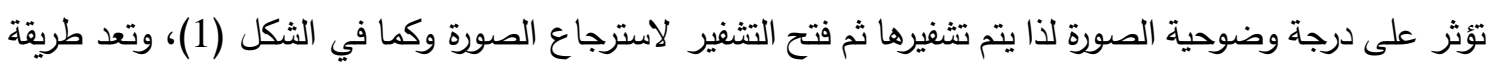
الكبس الحل الأمثل لتقليل حجم البيانات التي تتطلب لتمثيل الصورة الرقميه التي تكون على شكل مصفوفة مربعة

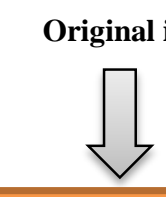

Encoder
Compressed image
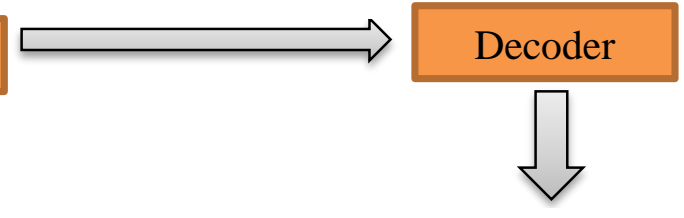

Reconstructed image

ثنائية الابعاد[2] [3]. 
إن كفاءة تقنيات وطرائق الكبس المختلفة أدت إلى استخدام الكبس في عدة مجالات وخاصة في مجال معالجة الصور، وقد تم إنجاز العديد من البحوث في هذا المجال في الدراسات السابقة. فقد قام عدد من الباحثين

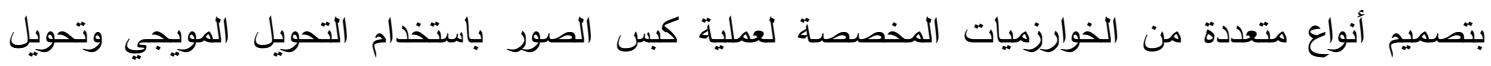
الكنتوري وتحويل الكيرفليت. في عام 2002 قد الباحث Minh N. Do أطروحته للدكتوراه التي تُعُدُ الأساس في لبعدي انطلاقة التحويلات الكنتورية حيث اعتمد الباحث طريقة جديدة وفعالة وذات خصائص لم توفر في أي تحويل آخر من تحويلات الصور المعروفة ومن تلك الخصائص الدقة المتعددة والاتجاهية المتعددة في التحليل [4]. في عام 2005 قدم Peter Michael, AhmedNabil بحثا بعنوان كبس الصور باستخدام التحويل الكنتوري وتم اجراء

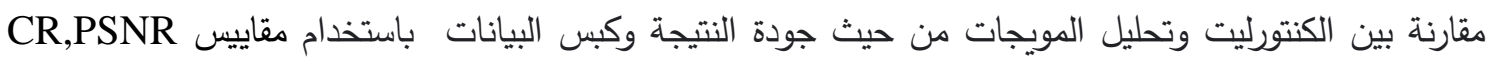

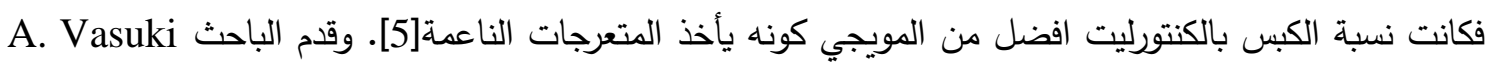
في عام 2009 بحثا بعنوان كبس الصور التدريجي باستخدام التحويل الكنتوري يعمل بكفاءة في التقاط المعلومات

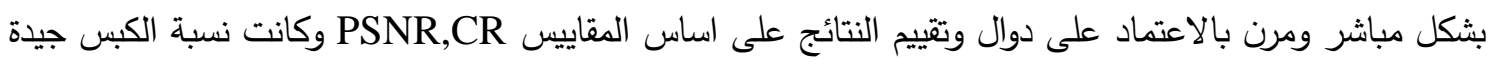

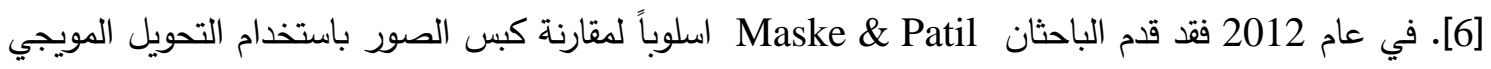
مع تحويل كيرفليت والارسال عبر التناة اللاسلكية وتقييم الاداء على اساس المقاييس المختارة فكانت نسبة الكبس

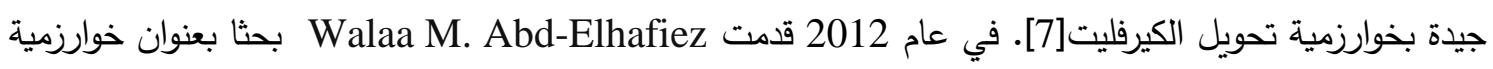
كبس الصور باستخدام تحويل الكيرفليت اعتمدت على تقسيم الصورة الى كتل من البكسل والاحتفاظ بعدد معين وحظر الباقي في DCT واعطت نتائج كبس جيدة بالاعتماد على مقاييس محددة[8]. في عام 2014 قدم Kumar Mathur و وكانت نتائج نسبة الكبس باستخدام تحويل المويجي افضل من DCT ,VQ [9]. في عام 2018 قدم

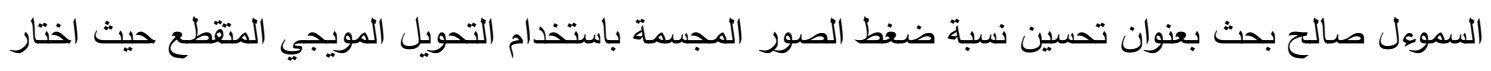

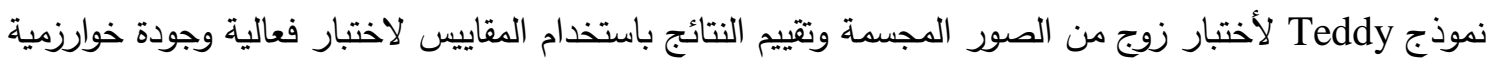
الكبس فحققت افضل نتيجة للصورة المسترجعة[17]. وفي عام 2018 قدم Khireddine بحثا بعنوان الاسلوب السريع والفعال لكبس الصور باستخدام التحويل الكيرفليت ومقارنة خوارزمية ترميز الصور التكيفية بالطرق

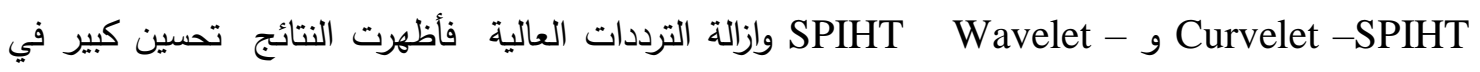
نسبة الكبس والاشارة الى الضوضاء العظمى[10]. قدم حسن ماهر نعمة في عام 2019 بحث بعنوان طريقة المهجنة لكبس الفيديو الرقمي باستخدام تقنيه التحويل المويجي وشجرة الصفر المويجية المطمورة] [18].

3. مشكلة البحث وأهدافه واهميته:

بناء على البحوث السابقة مشكلة البحث هي ازدياد التوجهات الحديثة في الآونة الاخيرة لاستخدام كبس

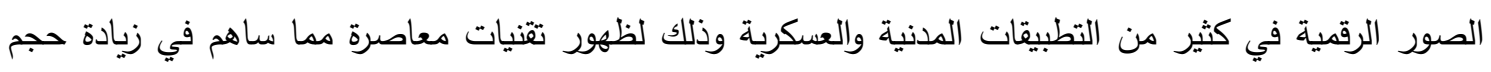

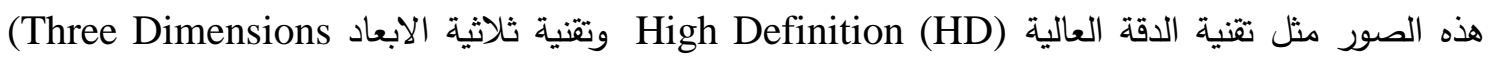
(3D)) لتخفيض تكاليف الخزن وتقليل وقت الارسال وتحافظ على جودة صورة. 
واحدى حلول هذه المشكلة هي دراسة تطبيق خوارزميات تحويلات (المويجي والكنتوري والكيرفليت) في تقنية كبس الصور الرقمية ومقارنة نسبة الكبس بين الخوارزميات للتحويلات (المويجي والكنتوري والكيرفليت) بالاعتماد على المقاييس (COR،CR،SNR،PSNR،MSE)، لغرض الوصول إلى أحسن النتائج في الاسترجاع الصورة والاحتفاظ بالمعالم الاساسية للصورة.

تستخدم تقنية كبس الصور لتوفير المساحة تعني توفير مساحة التخزين وتسهيل عملية نقلها وارسالها عبر وسائل وتقنيات الاتصال، ففي حال عدم استخدام تقنية كبس الصورة سوف نضطر لحجز مساحات كبيرة جداً لحفظ توفير

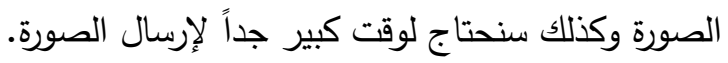
4. تقنيات كبس الصور يعرف الكبس بأنه تقنية تُستخدم لتقليل حجم ملف البيانات للصورة الرقمية لغرض المعالجة والخزن والنقل، مع الاحتفاظ بالمعلومات الضرورية للصورة [11]، وهنالك نوعان من الكبس:

\section{Lossless Compression الكبس بدون فقدان. . I}

في هذا النوع من الكبس من الممكن إعادة البيانات التي تم كبسها وبشكل مطابق للبيانات الأصلية، اي تسترد البيانات الاصلية للصورة بشكل دقيق بدون فقدان [12] [11]، ومن طرائق الكبس المستخدمة في هذا النوع:

• Huffamn Coding طريقة ترميز هوف مان

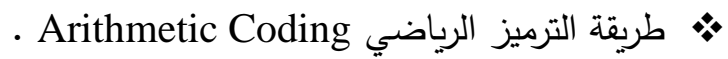

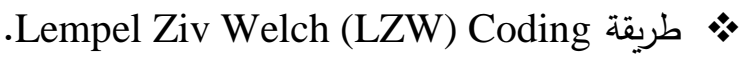

Lossy Compression الكبس بفقدان . .II

يتم في هذا النوع فقدان جزء من مجموعة البيانات للصورة الأصلية ولكن هذا الفقدان قد لا يؤثر بالدرجة الكبيرة على المعالم الرئيسية للصورة عند الاسترجاع، اي ان نسبة التثوه في الصورة قليل جدا وكلما كان التثوه اقل

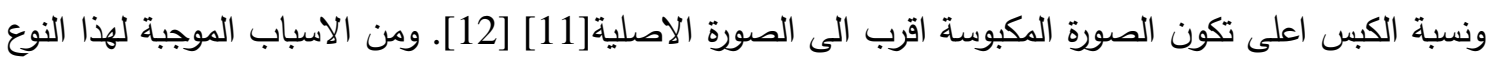

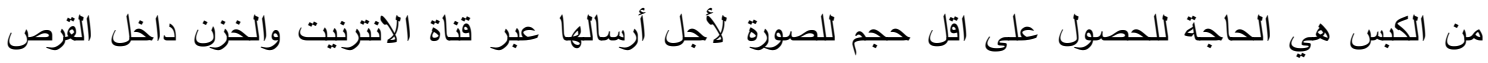
ومن طرائق الكبس المستخدمة في هذا النوع: • ثمويلات المويجية (Wavelet). • • ثشفير شجرة الصفر المويجية المطمورة (WZW).

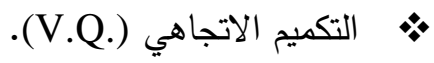

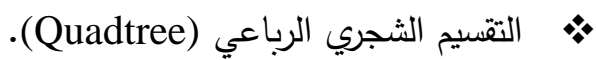
5. 5نهجية ومخطط البحث:

تم العمل في هذا البحث على تتفيذ خوارزميات التحويلات (المويجي والكنتوري والكيرفليت) لكبس صور الرقمية تتضمن عملية التتفيذ عدة مراحل وهي أدخال الصورة الرقمية، قراءة الصورة واجراء معالجة اولية، تحليل الصورة باستخدام الخوارزميات الثلاثة، تصفير الترددات العالية، استرجاع الصورة بعد الكبس ومن ثم الخطوة 
الاخيرة تطبيق المقاييس. كما في الثكل أدناه الذي يوضح المخطط العام للبحث في تقنية كبس الصور باستخدام

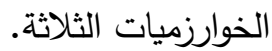

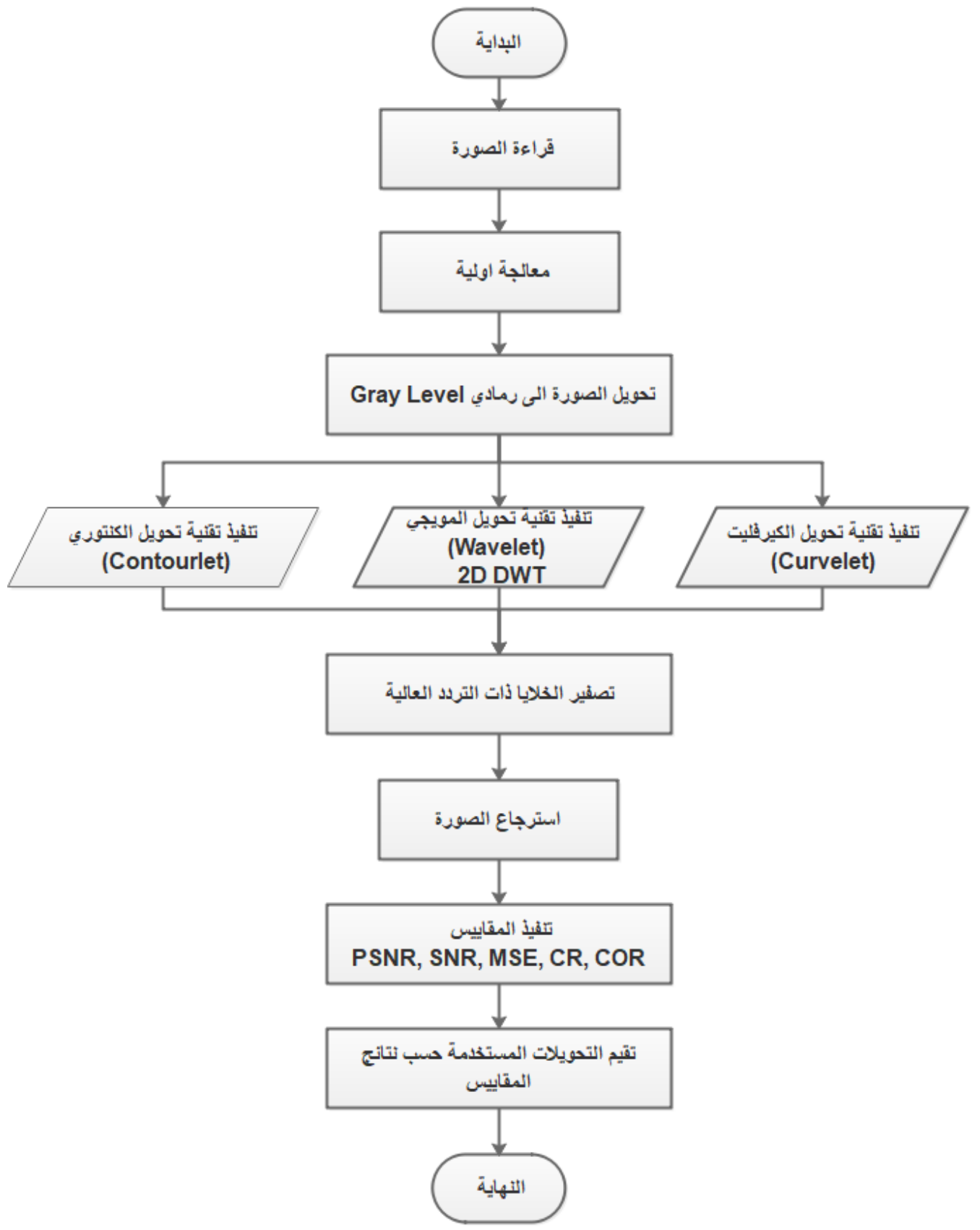

المخطط العام للبحث يوضح تقنية الكبس للصور باستخدام الخوارزميات الثلاثة

يعتبر التحويل المويجي المتقطع DWT 2D من التطبيقات المهمة التي تم استخدامها في معالجة الصور Image Compression and ( الرقمية (Image Processing) ومن هذه التطبيقات كبس الصور وتثفيرها Coding )، والذي يتعامل مع الصور من خلال التفاصيل التي تتمثل بها الصورة ويدعى بأسلوب التحليل متعدد

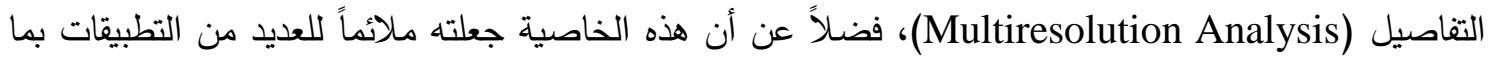
فيها معالجة صور الأقمار الصناعية وبياناتها في مجال التحس النائي. تمت عملية التحويل المويجي على هلى ملئه 
الصورة الداخلة الى حد مستويين (Two-Levels)، وإعتماد مويجة هار (Haar Wavelet) وطبقت على الصورة Lena

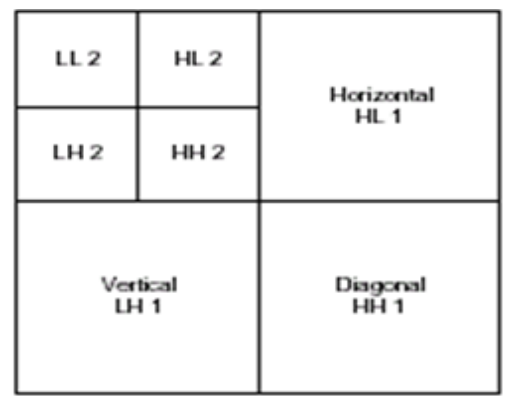

الثكل (2) هيكلية عمل تحويل المويجي المتقطع

التحويل الكنتوري يتعامل بكفاءة مع المتعرجات الناعمة Smooth Contours في الصور ، وهي تحويلات ثنائية البعد تعرف بصيغتها المتقطعة وذلك لالتقاط معلومات الحافات من جميع الاتجاهات، حيث تقوم التحويلات الكنتورية اولاً بتفكيك الصورة بواسطة Laplacian Pyramid Filter (LPF) ليتم تحديد الحافات بجميع المستويات، وبعد ذلك يطبق مرشح اتجاهي Direction filter bank (DFB) وذلك لإكمال الفراغات بين النقاط بهيكلية خطية. التحويلات الكنتورية تمتلك نفس الميزات في التحويلات المويجي من ناحية (تعدد المقياس، خاصية الزمن ويعرض درجة عالية من الاتجاهية والتوزيع الاتجاهي، أي ان الاختلاف الرئيسي بين الكنتورليت وأنظمة متعددة القياس أنه يسمح باختلاف ومرونة في عدد الاتجاهات في كل مقياس فضلا عن استعماله مجموعة المرشحات التكرارية (Iterated Filter Banks) الذي تجعله كفوءا على نحو حسابي [14]. يعد تحويل الكيرفليت امتدادا جديدا لتحويل المويجة والذي يهدف الى التعامل مع الظواهر المهمة التي تحدث على طول الحافات المتعرجة في الصور ثنائية الابعاد وينظر الية كتحويل هرمي متعدد المقياس، يمتلك تحويل الكيرفليت صفات الترددات الزمنية المكانية Time-Frequency Localization كصفات تحويل المويجة ولكنة يظهر بدرجة الصورة الرقمية، والحصول على تمثيل للحد الأقصى من المعلومات في الصورة [10].

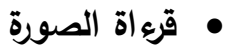
تم ادخال صورة (lena) القياسية الملونة من نوع (jpg) وبحجم (512×512) وتحويلها الى مصفوفة رقمية. وتحويل الصورة الملونة إلى صورة رمادية (Gray Level)، كذلك تتم المعالجة الاولية التحضيرية للشروع في عمليات المعالجة الاخرى مثل التحجيم، حيث تتم معالجة الصورة بأبعاد (256×256) كما في الثكل (3).
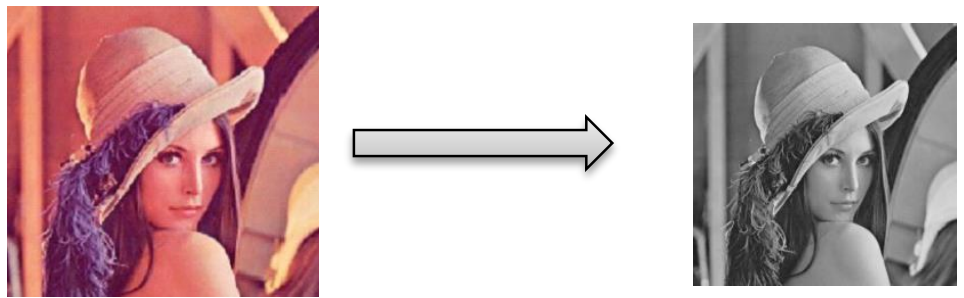

الشكل(3) يعرض صورة Lena قبل وبعد المعالجة 


\section{• تحليل الصورة باستخدام التحويلات الثلاثة (المويجي والكنتوري وإلكيرفليت)}

\section{>}

تحليل الصورة بحجم (256×256) باستخدام تحويل المويجي تم اختيار تقنية DWT2 باستخدام الفلتر Haar يكون تحليل اكثر كفاءة ودقة، فتتقسم الصورة الى اربع اجزاء (الجزء الاول هو مستوى الترددات الواطئة (LL) هو الجزء التقرببي Approximations الأكثر أهمية، ويعطي الصورة هويتها. اما الاجزاء الثلاثة الاخرى هم المستويات ذات الترددات العالية (HL,LH,HH) تعطي الصورة تفاصيلها الدقيقة

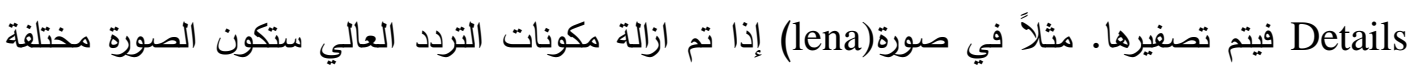
ولكن واضحة المعالم، واذا تم ازالة المستوى ذات التردد الواطئ تكون الصورة مشوهة وغير واضحة. الثكل (4) يوضح تحليل صورة باستخدام خوارزمية التحويل المويجي [15]. التحويل الكنتوري:

تم تحليل الصورة باستخدام التحويلات الكنتورية وبالاعتماد على معاملات الكنتورليت ذات الترددات

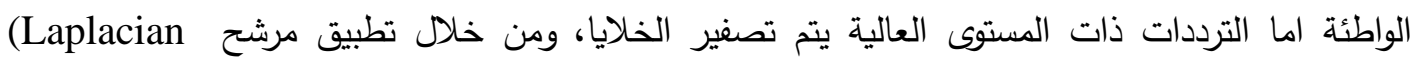
على الصورة الأصلية حيت يتم تحديد الحافات بجميع المستويات وتطبيق المرشح الاتجاهي وذلك لإكمال الفراغات بين النقاط بهيكلية خطية على الصورة Direction filter bank (DFB)

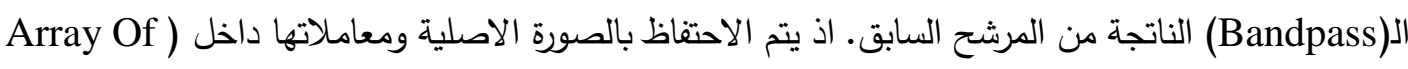
(Cells اذ ان كل عنصر من عناصر هذه المصفوفة هو عبارة عن خلية تحتوي على واحدة او اكثر من المصفوفات ثنائية البعد، والتي تمثل كل واحدة منها احد معاملات التي تم الحصول عليها من الكنتورليت

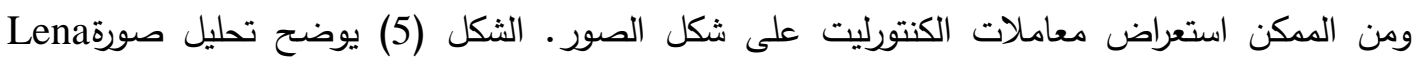

باستخدام خوارزمية تحويل الكنتوري [14].

\section{>}

عند تطبيق تحويل الكيرفليت المتقطع السريع باستخدام تقنية FDCT-WRAPPING التغليف للتحليل متعدد المقياس يتم تقسيم الصورة الى سلسلة من الحزم غير مرتبطة والتي تتكون منها معاملات كيرفليت. في

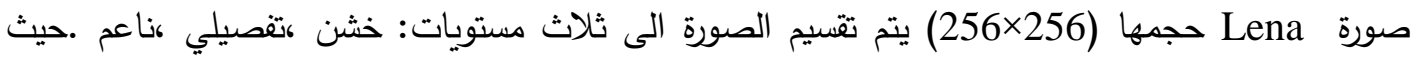

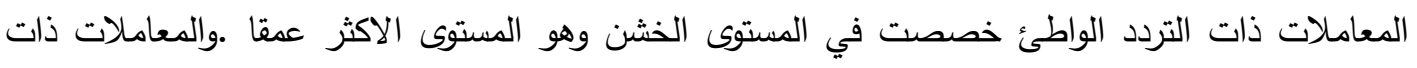
الترددات العالية خصصت في المستوى الناعم والذي هو المستوى الخارجي، والمعاملات ذات التردئ التردات

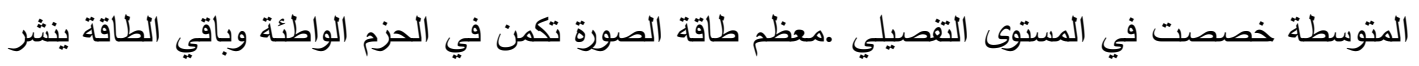

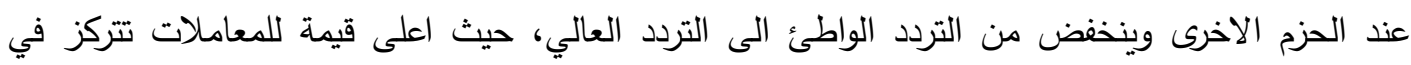

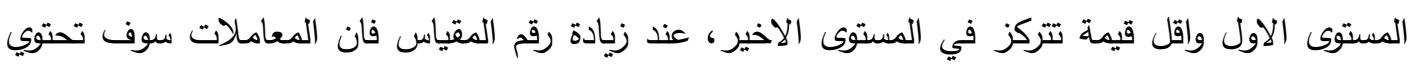

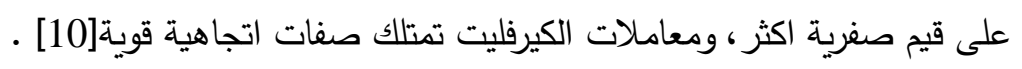

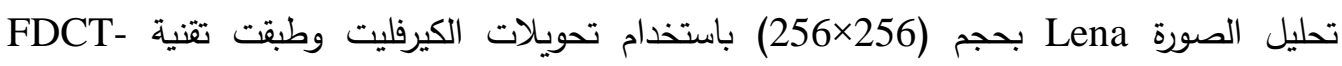
WRAPPING بالصورة الاصلية ومعاملاتها داخل (Array Of Cells) اذ ان كل عنصر من عناصر هذه المصفوفة هو 
عبارة عن خلية تحتوي على واحدة او أكثر من المصفوفات ثنائية البعد، والتي تمثل كل واحدة منها أحد معاملات التي تم الحصول عليها من الكيرفيلت ومن المكن استعراض معاملات الكتئ الكيرفليت على شكل الصور . الثكل (6) يوضح تحليل صورةLena باستخدام خوارزمية تحويل الكيرفيلت. • تصفير الصورة باستخدام تحويلات

> تصفير معاملات الصورة باستخدام تحويل المويجي

نأخذ جزء التقريبي Approximations للمستوى الاول والثاني (هي ترددات ذات المستويات الواطئة (LH1, ويتم تصفير جزء Details للمستوى الاول والثاني (هي ترددات ذات المستويات العالية لهردية (LL1 . Lena وطبقت على صورة HL1, HH1 > تصفير معاملات الصورة باستخدام تحويل الكنتوري في هذه الخطوة يتم اخذ الخلايا ذات الترددات الواطئة، اما الخلايا ذات المستويات العالية يتم تصفيرها لان تحتوي على ضوضاء وتكون اقل اهمية، وهذا الكلام ينطبق على تصفير معاملات الصورة باستخدام تحويل الكيرفليت.

\section{• استرجاع الصورة للتحويلات (المويجي والكنتوري والكيرفليت)}

عملية استرجاع الصورة لجزء (Approximations) هي معاكسة لعملية كبسها حيث أن عملية الكبس

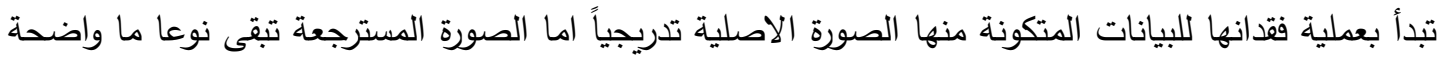
المعالم، والغاية الاساسية من عملية الكبس الحصول على صورة واضحة المعالم وبأقل حجم ممكن وتقليل الزمن اللازم للتنفيذ بالإسراع من عملية نقل المعلومات. الاشكال التالية توضح عملية تحليل صورة Lena لخوارزميات تحويلات الثلاثة (المويجي والكنتوري وكيرفليت) 

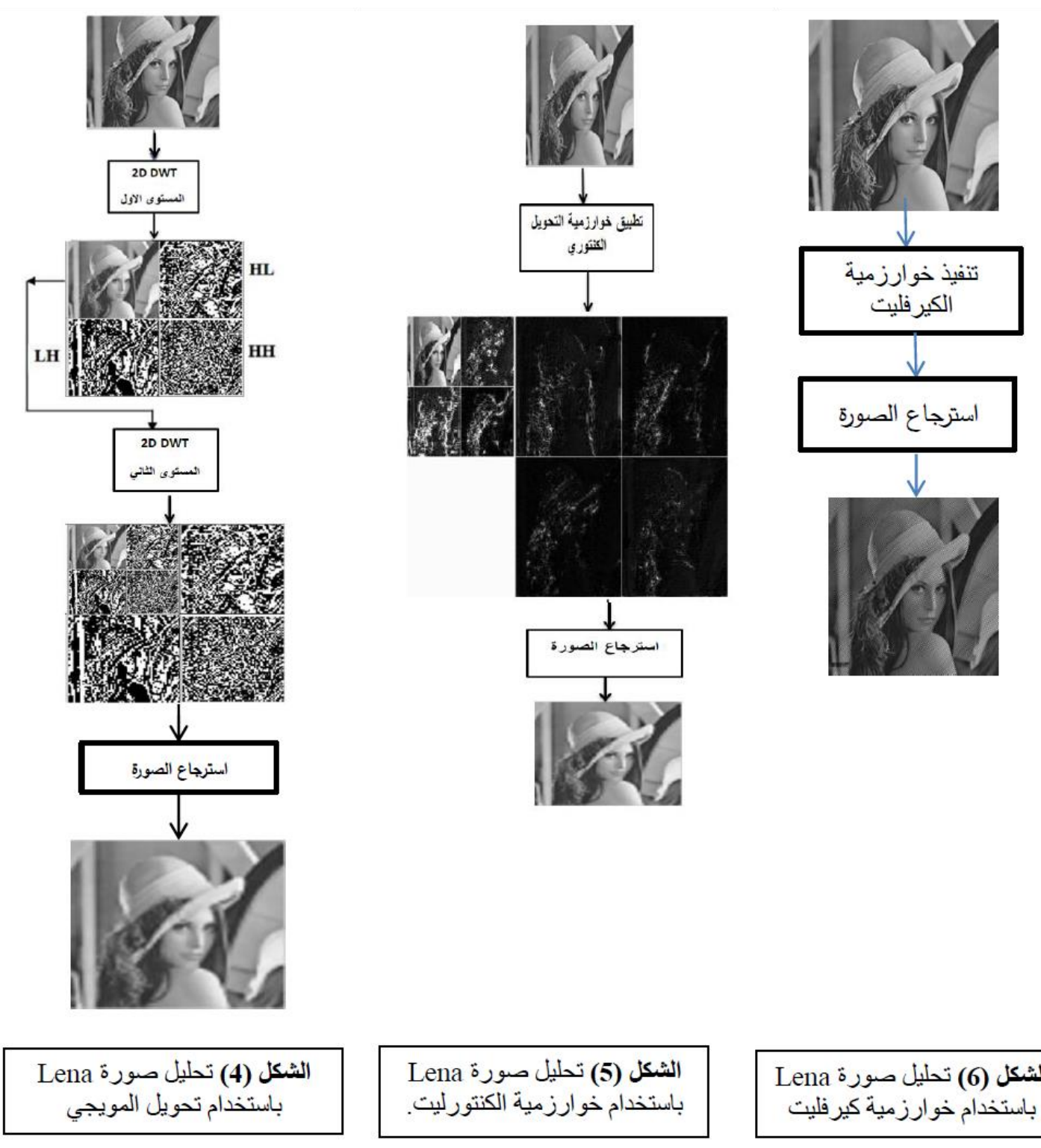
الثكل (5) تحليل صورة Lena

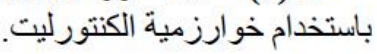

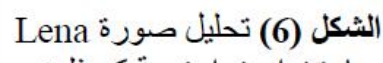

باستخدام خوارزمية كيرفليت

• • قياس كفاءة اداء الخوارزميات

هنالك انواع كثيرة من المقاييس التي تستخدم مع عمليات الكبس لقياس نسبة الكبس ونسبة الضوضاء والى هذ

اخره من المقاييس وفي هذا البحث استخدمنا بعض انواع هذه المقاييس ومنها:

MSE(mean sequence error) مقياس متوسط الخطأ التربيعي *

إن قيمة متوسط الخطأ التربيعي تثير الى مقياس الخطأ بين قيم نقاط الصورة الأصلية(غير المكبوسة) وقيم نقاط الصورة الناتجة (بعد الكبس)، وكلما انخفضت قيمة متوسط الخطأ التربيعي حصلنا على نتائج افضل

$M S E=\frac{\sum_{\mathrm{m}, \mathrm{n}}[11(\mathrm{~m}, \mathrm{n})-\mathrm{I} 2(\mathrm{~m}, \mathrm{n})]^{2}}{\mathrm{M} * \mathrm{~N}}$

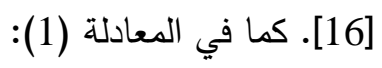


Peak signal to noise ratio(PSNR) مقياس نسبة الاثارة الى الضوضاء نسبة الاشارة الى الضوضاء العظمى كلما اقتربت القيمة من الصفر كلما كانت الضوضاء في الاشارة

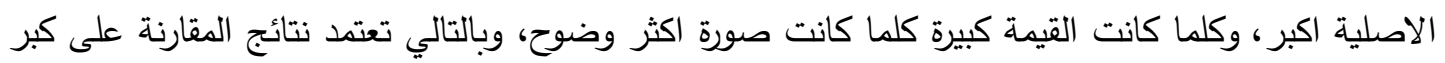
القيمة ال PSNR [16] كما في المعادلة (2): $P S N R=10 \log 10\left(M A X i^{2} / M S E\right)$

SNR (signal to noise ratio) نسبة الاشارة الى الضوضاء

هو مقياس يستخدم في العلوم والهندسة يقارن بين الإثارة والضوضاء[16]. كما في معادلة (3) $S N R=10 \log 10\left[\frac{\sigma y^{2}}{\sigma x^{2}}\right]$.

\section{Normalization correlation (NC) ترابط التربيعي *}

يعتبر معامل الارتباط مقياسا لمدى تثابه بين المتغيرين المستقلين، يقيس هذا المعامل الفرق بين الصورة

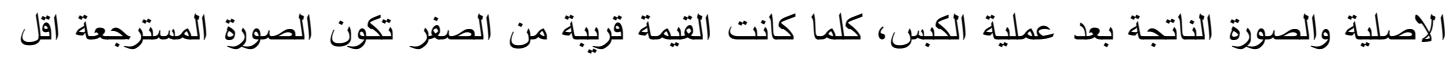
تطابق او ارتباط بالصورة الاصلية، وكلما كانت القيمة قريبة من الواحد تكون الصورة المسترجعة مطابقة

$$
\operatorname{corr}=\frac{\sum_{m} \sum_{n}\left(\boldsymbol{A}_{m n}-\bar{A}\right)\left(B_{m n}-\bar{B}\right)}{\sqrt{\left(\sum_{m} \sum_{n}\left(A_{m n}-\bar{A}\right)^{2}\right)\left(\sum_{m} \sum_{n}\left(\boldsymbol{B}_{m n}-\bar{B}\right)^{2}\right)}}
$$

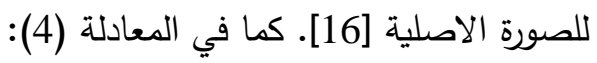

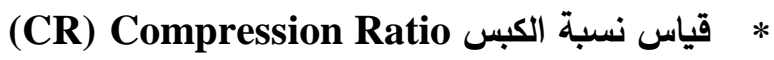

تتناسب نسبة الكبس علاقة عكسية مع جودة الصورة كلما كانت نسبة الكبس كبيرة كلما كانت جودة الصورة المسترجعة اقل [16].

نسبة الكبس(CR)= الصورة الاصلية/ الصورة المكبوسة

7. - النتائج والمناقشة:

Lena النتائج التالية بعد تتفيذ خوارزميات التحويلات الثلاثة (المويجي والكنتوري والكيرفليت) لكبس صورة حصلنا على نتائج حيث تم تطبيق المقاييس الخمسة المستخدمة بالبحث التي هي (PSNR, SNR, MSE, COR

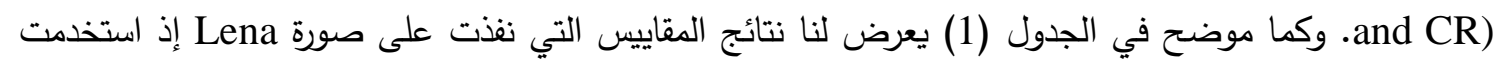
لتحديد جودة الصورة المسترجعة بعد الكبس باستخدام الخوارزميات (للتحويل المويجي والكنتوري والكيرفليت) ومن فئن خلال هذه النسب تم التعرف على اكفأ خوارزمية لكبس الصورة الرقمية من بين الخوارزميات المستخدمة . جدول (1) يعرض المقاييس بعد كبس صورة Lena

\begin{tabular}{|c|c|c|c|c|c|}
\hline Transforms & MSE & PSNR & SNR & COR & CR \\
\hline Wavelet & 8.3228 & 43.3509 & 11.6841 & 0.9645 & 16 \\
\hline Contoulet & 8.1402 & 43.5436 & 11.5077 & 0.9074 & 16 \\
\hline Curvelet & 18.6390 & 36.3478 & 17.5921 & 0.9043 & 60.1800 \\
\hline
\end{tabular}


الثكل (7) يوضح نسب المقاييس (PSNR, SNR, MSE, COR and CR) بعد استخدام الخوارزميات التحويلات (المويجي والكنتوري والكيرفليت) لتتفيذ عملية الكبس على الصورة الرقمية (Lena) تم تحديد افضل خوارزمية كفوءة في عملية الكبس هي خوارزمية التحويل كيرفليت عن غيرها من الخوارزميات استتادا على المقاييس (PSNR, SNR, MSE, COR and CR)
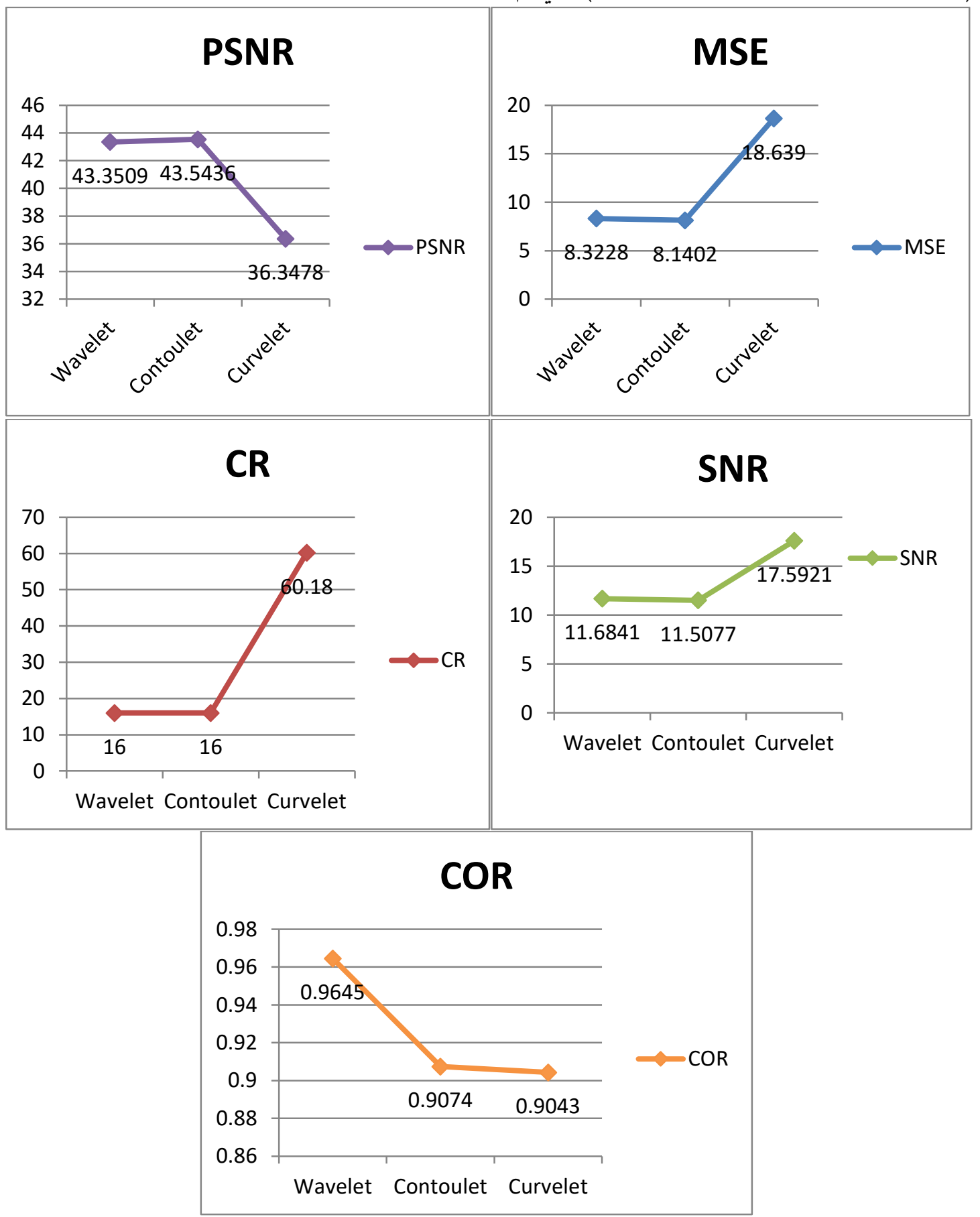

الثكل (7) يمثل نسب المقاييس (PSNR,SNR,MSE,COR,CR) التي استخدمت لكبس صورة Lena مقارنة مع الطرق السابقة تم الحصول على نتائج افضل من الباحث سمؤل صالح في خوارزمية التحويل المويجي حيث كانت قيمة نسبة الكبس Mridul Kumar وقق نسبة كبس CR (1.1247) والباحث 
(10.765)، بالنسبة لخوارزمية التحويل الكنتوري حقق الباحث Ahmed Nabil نسبة كبسCR (3.1 )، واخيرا خوارزمية التحويل الكيرفليت حققت الباحثة Walaa M. Abd-Elhafiez نسبة كبس CR (13.884) والباحث CR حقق نسبة كبس CR Peter Michael GOEBEL في خوارزمية التحويل المويجي (16)، وخوارزمية التحويل الكنتوري (16)، وخوارزمية تحويل الكيرفليت(60.18).

8.

تم في هذا البحث تطبيق خوارزميات التحويلات (المويجي، الكنتوري، الكيرفليت) على الصورة القياسية ل

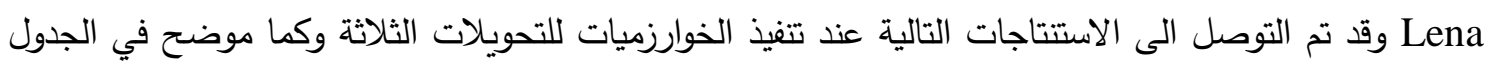

$$
\text { (1) والثكل (7) تظهر المقاييس وكالتالي: }
$$

1. مقياس PSNR نسبة الاثارة الى الضوضاء العظمى في خوارزمية التحويل كيرفليت اقل من خوارزميتي

$$
\text { التحويل المويجي وبعدها الكنتوري. }
$$

2. مقياس MSE نسبة الخطأ التربيعي في خوارزمية التحويل الكنتوري اقل مما هو علية في خوارزميتي التحويل

$$
\text { المويجي وبعدها الكيرفليت. }
$$

3. مقياس SNR نسبة الاشارة الى الضوضاء في خوارزمية التحويل الكنتوري اقل من خوارزميتي التحويل المويجي

$$
\text { وبعدها الكيرفليت. }
$$

4. مقياس COR قياس نسبة التطابق في خوارزمية التحويل الكنتوري أعطت اعلى نسبة تطابق بين الصورة الاصلية والصورة المسترجعة وبعدها خوارزميتي التحويل المويجي وبعدها الكيرفليت.

5. المقياس CR لقياس نسبة الكبس الصورة الاصلية على الصورة المكبوسة بعد تنفيذ الخوارزميات الثلاثة (للتحويل المويجي والكنتوري والكيرفليت) لكبس الصور الرقمية، حيث أن نتيجة المقياس باستخدام خوارزمية

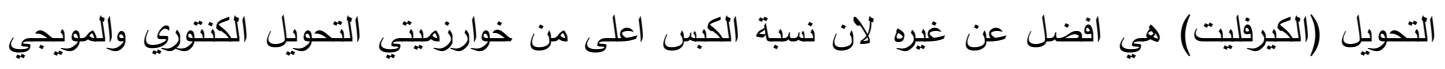
ولكن على حساب جودة الصورة.

9. - 9 الاعمال المستقبلية

إن افكار ونتائج تطبيق خوارزميات هذا البحث يمكن أن تتطور لتشمل العديد من الاعمال المستقبلية للبحث

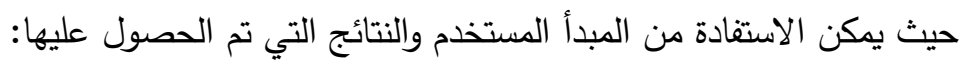
1- امكانية تطبيق هذه الخوارزميات في مجال كبس الفيديو وكبس الصوت لما لما له من أهمية كبيرة.

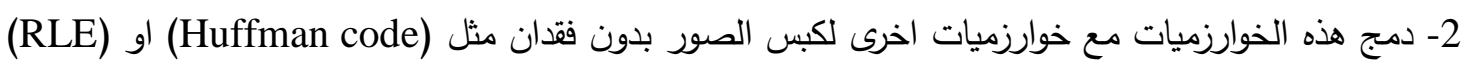

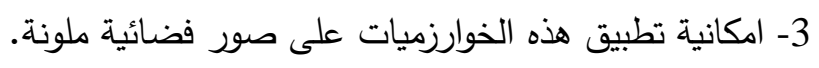

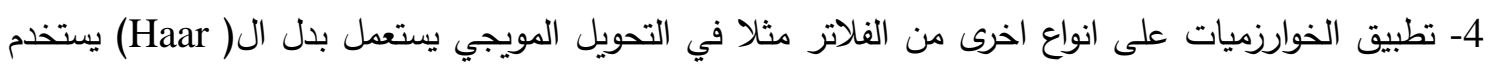

5- تطبيق خوارزميات التحسين على الصورة المكبوسة للحصول على صورة ذات جودة عالية.

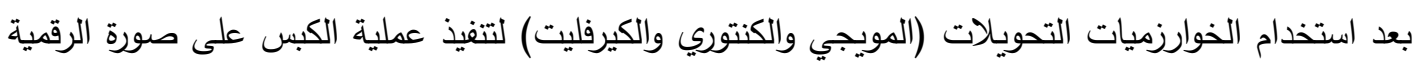
تم تحديد اكثر خوارزمية كفوءة في عملية الكبس هي خوارزمية التحويل كيرفليت عن غيرها من الخوارزميات استتادا على الدقاييس (PSNR,SNR,MSE,COR,CR) التي تم حسابها بعد عملية كبس الصور 


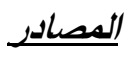

[1] Gonzalez, R.C., R.E. Woods, and S.L. Eddins, Digital image processing using MATLAB. 2004: Pearson Education India.

[2] Gonzales, R.C. and R.E. Woods, Digital image processing. 2002, Prentice hall New Jersey.

[3] Tan, C.L., Still image compression using wavelet transform. The University of Queeslands, 2001.

[4] Minh, N., Directional multiresolution image representations. University of Canberra, 2002.

[5] Belbachir, A.N. and P. Göbel, The contourlet transform for image compression. 2005: na.

[6] Vasuki, A. and P. Vanathi, Progressive image compression using contourlet transform. International Journal of Recent Trends in Engineering, 2009. 2(5): p. 193.

[7] Maske, N.D. and W.V. Patil, Comparison of image compression using wavelet for curvelet transform \& transmission over wireless channel. International Journal of Scientific and Research Publications, 2012. 2(5): p. 1-5.

[8] Abd-Elhafiez, W.M., Image compression algorithm using a fast curvelet transform. International Journal of Computer Science and Telecommunications, 2012. 3(4): p. 43.47.

[9] Mathur, G., R. Mathur, and M.K. Mathur, A comparative study of various lossy image compression techniques. ETRASCT. 14: p. 165-169.

[10] Inouri, L., et al., A fast and efficient approach for image compression using curvelet transform. Sensing and Imaging, 2018. 19(1): p. 26.

[11] Hilles, S.M. Sofm And Vector Quantization For Image Compression By Component. in 2018 International Conference on Smart Computing and Electronic Enterprise (ICSCEE). 2018. IEEE.

[12] Fisher, R.B., et al., Dictionary of computer vision and image processing. 2013: John Wiley \& Sons.

[13] Deepa, M., Wavelet and curvelet based thresholding techniques for image denoising. International Journal of Advanced Research in Computer Science and Electronics Engineering (IJARCSEE), 2012. 1(10): p. 10.

[14] Do, M.N. and M. Vetterli, The contourlet transform: an efficient directional multiresolution image representation. IEEE Transactions on image processing, 2005. 14(12): p. 2091-2106.

[15] Hasan, T.S., Image compression using discrete wavelet transform and discrete cosine transform. Journal of Applied Sciences Researches, 2017. 13: p. 1-8.

[16] Thirumoorthi, C. and T. Karthikeyan, A study on discrete wavelet transform compression algorithm for medical images. 2017. 
[17 [ ساعي, صالح, السموءل, \& al al., (2018) حاتم. (2018). تحسين نسبة كبس الصور المجسمة ISSN: 2079-3081, 39(3). سلسلة العلوم .SGM باستخدام التحويل المويجي المتقطع النعمة, ح. م. أ. (2019). طريقة مهجنة لكبس الفيديو الرقمي باستخدام تقنية التحويل المويجي وشجرة الصفر المويجية المطمورة. مجلة التربية والعلم, 28(1), 211-225. 